Advances in Dynamical Systems and Applications.

ISSN 0973-5321 Volume 16, Number 1 (2021), pp. 355-368

(C) Research India Publications

https://dx.doi.org/10.37622/ADSA/16.1.2021.355-368

\title{
On the dynamics of the singularly perturbed Riccati differential equation with two different delays
}

\author{
A.M.A. EL-Sayed ${ }^{1}$, S.M. Salman ${ }^{2}$, S.Ramadan*3 \\ ${ }^{1}$ Affiliation for Faculty of Science, Mathematics Department, Alexandria University \\ ${ }^{2}$ Affiliation for Faculty of Education, Mathematics Department, Alexandria University \\ ${ }^{3}$ Affiliation for Faculty of Science, Mathematics Department, Alexandria University
}

\begin{abstract}
In this paper, we consider the singularly perturbation of the Riccati difference equation with two different delays. At first, we study the local stability of the fixed points and its corresponding characteristic equation of the linearized system. At second, we show that there is Hopf bifurcation with restricted condition for occurrence. Then we get out the discretized system by applying the method of steps. Local stability and bifurcation analysis of the discretized system. We compare the results with the results of the Riccati differential equation with two different delays. Finally, numerical simulations including bifurcation diagram, Lyapunov exponent and phase portraits are carried out to confirm the analytical findings .
\end{abstract}

Keywords: Singular perturbation, Differential delay, Fixed points, Chaos, Bifurcation.

\section{INTRODUCTION}

The ordinary differential equation involving at least one delay term and the highest derivative is multiplied by a small parameter namely singularly perturbed delay differential equation $[1,2,3,4,10,8,6]$. In recent decades, the analysis and design

\footnotetext{
${ }^{*}$ Corresponding author.
} 
theories for singularly perturbed systems with time-delay have been of considerable concern. For example, Fridman has considered the effect of small delay on stability of the singularly perturbed systems $[7,12,15]$. Two approaches to the asymptotic analysis and solution of this problem are proposed. In the first approach, an asymptotic solution of the singularly-perturbed system of functional-differential equations of Riccati type, associated with the original problem by the sufficient conditions of the existence of its solution, is constructed[13, 14]. Based on this asymptotic solution, conditions for the existence of a solution of the original problem, independent of the small parameter of singular perturbations, are derived $[5,16]$.

In this paper, we will discuss the dynamic behavior of the singularly perturbed Riccati differential equation with two different delays given in the form

$$
\begin{array}{cr}
\epsilon \frac{d x}{d t}=-x(t)+1-\rho x(t-1) x(t-2), & t \in[0, T], \\
x(t)=x_{0}, & t \leq 0 .
\end{array}
$$

\subsection{Stability and bifurcation}

Consider the problem (1). Solving the equation ([11])

$$
-x+1-\rho x^{2}=0,
$$

we obtain the two fixed points,

$$
\left(x_{1,2}\right)^{*}=\left(\frac{-1}{2 \rho}\right)(1 \pm \sqrt{1+4 \rho}) .
$$

At the neighborhood of $\left(x_{1}\right)^{*}$ the linearized equation is

$$
\epsilon \frac{d y}{d t}=-y(t)+\frac{1}{2}(1+\sqrt{1+4 \rho}) y(t-1)+\frac{1}{2}(1+\sqrt{1+4 \rho}) y(t-2)
$$

where

$$
y(t)=y(t)-\left(\left(\frac{-1}{2 \rho}\right)(1+\sqrt{1+4 \rho})\right) .
$$

Then the characteristic equation is

$$
\lambda+\frac{1}{\epsilon}-\frac{1}{2 \epsilon}(1+\sqrt{1+4 \rho}) e^{-\lambda}-\frac{1}{2 \epsilon}(1+\sqrt{1+4 \rho}) e^{-2 \lambda}=0 .
$$

Theorem 1. When the parameter $\rho$ passes through the critical value $\rho=\rho_{*}=$ $\frac{1}{4}\left[\left(\frac{1+\epsilon^{2} \omega_{0}^{2}-\left(\cos \left(\omega_{0}\right)+\epsilon \omega_{0} \sin \left(\omega_{0}\right)\right.}{\cos \left(\omega_{0}\right)+\epsilon \omega_{0} \sin \left(\omega_{0}\right)}\right)^{2}-1\right], \omega_{0}=\tan \left(2 \omega_{0}\right)\left(1-s \cos \left(\omega_{0}\right)\right)+s \sin \left(\omega_{0}\right)$, there is Hopf bifurcation from the equilibrium $\left(x_{1}\right)^{*}=\left(\frac{-1}{2 \rho}\right)(1+\sqrt{1+4 \rho})$ to a periodic orbit. 
Proof. Let $\lambda=i \omega_{0}, \omega_{0} \in R^{+}$be a for (3) for $\rho=\rho_{*}$, then we obtain

$$
\begin{gathered}
i \epsilon \omega_{0}+1-\frac{1}{2}\left(1+\sqrt{1+4 \rho_{*}}\right) e^{-i \omega_{0}}-\frac{1}{2}\left(1+\sqrt{1+4 \rho_{*}}\right) e^{-2 i \omega_{0}}=0, \\
\text { then, } 1-\frac{1}{2}\left(1+\sqrt{1+4 \rho_{*}}\right) \cos \left(\omega_{0}\right)-\frac{1}{2}\left(1+\sqrt{1+4 \rho_{*}}\right) \cos \left(2 \omega_{0}\right)=0, \\
\epsilon \omega_{0}-\frac{1}{2}\left(1+\sqrt{1+4 \rho_{*}}\right) \sin \left(\omega_{0}\right)-\frac{1}{2}\left(1+\sqrt{1+4 \rho_{*}}\right) \sin \left(2 \omega_{0}\right)=0, \\
\text { let } \frac{1}{2}\left(1+\sqrt{1+4 \rho_{*}}\right)=s \\
\text { then, } 1-s \cos \left(\omega_{0}\right)-s \cos \left(2 \omega_{0}\right)=0 \\
\epsilon \omega_{0}-s \sin \left(\omega_{0}\right)-s \sin \left(2 \omega_{0}\right)=0 .
\end{gathered}
$$

By solving equation (4) and equation (5) We get,

$$
\begin{gathered}
s=\frac{1+\epsilon^{2} \omega_{0}^{2}}{2\left(\cos \left(\omega_{0}\right)+\epsilon \omega_{0} \sin \left(\omega_{0}\right)\right)} . \\
\text { Then, } \rho_{*}=\frac{1}{4}\left[\left(\frac{1+\epsilon^{2} \omega_{0}^{2}-\left(\cos \left(\omega_{0}\right)+\epsilon \omega_{0} \sin \left(\omega_{0}\right)\right.}{\cos \left(\omega_{0}\right)+\epsilon \omega_{0} \sin \left(\omega_{0}\right)}\right)^{2}-1\right],
\end{gathered}
$$

to get $\omega_{0}$,

$$
\begin{array}{r}
\frac{\epsilon \omega_{0}-s \sin \left(\omega_{0}\right)}{1-s \cos \left(\omega_{0}\right)}=\frac{\sin \left(2 \omega_{0}\right)}{\cos \left(2 \omega_{0}\right)}, \\
\omega_{0}=\frac{1}{\epsilon}\left[\tan \left(2 \omega_{0}\right)\left(1-s \cos \left(\omega_{0}\right)\right)+s \sin \left(\omega_{0}\right)\right] .
\end{array}
$$

Now, we are left with the condition $\left.\frac{d(\operatorname{Re}(\lambda))}{d \rho}\right|_{\rho} \neq 0$. To show that this condition is satisfied,

let $\lambda=k(\rho)+i \omega(\rho)$ and using equation (3),

we get,

$$
\epsilon(k+i \omega)+1-\frac{1}{2}(1+\sqrt{1+4 \rho}) e^{-k-i \omega}-\frac{1}{2}(1+\sqrt{1+4 \rho}) e^{-2(k+i \omega)}=0,
$$


then, we have,

$$
\begin{gathered}
\epsilon k+1-\frac{1}{2}(1+\sqrt{1+4 \rho}) e^{-k} \cos (\omega)-\frac{1}{2}(1+\sqrt{1+4 \rho}) e^{-2 k} \cos (2 \omega)=0 \\
\epsilon \omega+\frac{1}{2}(1+\sqrt{1+4 \rho}) e^{-k} \sin (\omega)+\frac{1}{2}(1+\sqrt{1+4 \rho}) e^{-2 k} \sin (2 \omega)=0 .
\end{gathered}
$$

differentiate equation(6) and equation (7) with respect to $\rho$, we get,

$$
\begin{aligned}
& \epsilon \frac{d k}{d \rho}+ \frac{1}{2} e^{-k} \cos (\omega) \frac{d k}{d \rho}+\frac{1}{2} e^{-k} \sin (\omega) \frac{d \omega}{d \rho} \\
&-\frac{1}{2} e^{-k} \cos (\omega) \frac{4}{2 \sqrt{1+4 \rho}}+\frac{1}{2}(\sqrt{1+4 \rho}) e^{-k} \cos (\omega) \frac{d k}{d \rho} \\
&+\frac{1}{2}(\sqrt{1+4 \rho}) e^{-k} \sin (\omega) \frac{d \omega}{d \rho}+e^{-2 k} \cos (2 \omega) \frac{d k}{d \rho} \\
&+e^{-2 k} \sin (2 \omega) \frac{d \omega}{d \rho}+(\sqrt{1+4 \rho}) e^{-2 k} \cos (2 \omega) \frac{d k}{d \rho} \\
&+(\sqrt{1+4 \rho}) e^{-2 k} \sin (2 \omega) \frac{d \omega}{d \rho}-\frac{1}{2} e^{-2 k} \frac{4}{2 \sqrt{1+4 \rho}} \cos (2 \omega)=0 \\
&=\frac{d k}{d \rho}\left(\epsilon+\frac{1}{2} e^{-k} \cos (\omega)+\frac{1}{2}(\sqrt{1+4 \rho}) e^{-k} \cos (\omega)+e^{-2 k} \cos (2 \omega)+(\sqrt{1+4 \rho}) e^{-2 k} \cos (2 \omega)\right) \\
&+\frac{d \omega}{d \rho}\left(\frac{1}{2} e^{-k} \sin (\omega)+\frac{1}{2}(\sqrt{1+4 \rho}) e^{-k} \sin (\omega)+e^{-2 k} \sin (2 \omega)+(\sqrt{1+4 \rho}) e^{-2 k} \sin (2 \omega)\right) \\
&-\frac{e^{-k} \cos (\omega)}{\sqrt{1+4 \rho}}-\frac{e^{-2 k} \cos (2 \omega)}{\sqrt{1+4 \rho}}=0
\end{aligned}
$$

$$
\begin{aligned}
\epsilon \frac{d \omega}{d \rho} & -\frac{1}{2} e^{-k} \sin (\omega) \frac{d k}{d \rho}+\frac{1}{2} e^{-k} \cos (\omega) \frac{d \omega}{d \rho} \\
& +\frac{1}{2} e^{-k} \sin (\omega) \frac{4}{2 \sqrt{1+4 \rho}}-\frac{1}{2}(\sqrt{1+4 \rho}) e^{-k} \sin (\omega) \frac{d k}{d \rho} \\
& +\frac{1}{2}(\sqrt{1+4 \rho}) e^{-k} \cos (\omega) \frac{d \omega}{d \rho}-e^{-2 k} \sin (2 \omega) \frac{d k}{d \rho} \\
& +e^{-2 k} \cos (2 \omega) \frac{d \omega}{d \rho}-(\sqrt{1+4 \rho}) e^{-2 k} \sin (2 \omega) \frac{d k}{d \rho} \\
& +(\sqrt{1+4 \rho}) e^{-2 k} \cos (2 \omega) \frac{d \omega}{d \rho}+\frac{1}{2} e^{-2 k} \frac{4}{2 \sqrt{1+4 \rho}} \sin (2 \omega)=0
\end{aligned}
$$




$$
\begin{aligned}
& =\frac{d k}{d \rho}\left(-\frac{1}{2} e^{-k} \sin (\omega)-\frac{1}{2}(\sqrt{1+4 \rho}) e^{-k} \sin (\omega)\right. \\
& \left.-e^{-2 k} \sin (2 \omega)-(\sqrt{1+4 \rho}) e^{-2 k} \cos (2 \omega)\right) \\
& +\frac{d \omega}{d \rho}\left(\epsilon+\frac{1}{2} e^{-k} \cos (\omega)+\frac{1}{2}(\sqrt{1+4 \rho}) e^{-k} \cos (\omega)\right. \\
& \left.+e^{-2 k} \cos (2 \omega)+(\sqrt{1+4 \rho}) e^{-2 k} \cos (2 \omega)\right) \\
& +\frac{e^{-k} \sin (\omega)}{\sqrt{1+4 \rho}}+\frac{e^{-2 k} \sin (2 \omega)}{\sqrt{1+4 \rho}}=0 .
\end{aligned}
$$

Solving equation (8) and equation (9) for $\frac{d k}{d \rho}$, we obtain

$$
\left.\frac{d(\operatorname{Re}(\lambda))}{d \rho}\right|_{\rho=\rho_{*}}=\left.\frac{d k}{d \rho}\right|_{k=0, \omega=\omega_{0}, \rho=\rho_{*}} \neq 0
$$

\subsection{The discretized system}

By using the method of steps, we can get out the discrete-time version of The system (1) by the following steps, the system can be written as ([12])

$$
\begin{array}{r}
\epsilon \frac{d x}{d t}=-x(t)+1-\rho x(t-1) y(t-1), \\
y(t)=x(t-1), \\
x(t)=x_{0}, t \leq 0 .
\end{array}
$$

The discretized model of the system (1) is obtained via the method of steps as follows let $t \in(0,1]$, then,

$$
\begin{gathered}
y_{1}(t)=x_{0}, \\
x_{1}(t)=x_{0} e^{\frac{-t}{\epsilon}}+\frac{1}{\epsilon} \int_{0}^{t} e^{\frac{s-t}{\epsilon}}(1-\rho x(s-1) y(s-1)) d s, \\
x_{1}(t)=x_{0} e^{\frac{-t}{\epsilon}}+\left(1-\rho x_{0} y_{1}\right)\left(1-e^{\frac{-t}{\epsilon}}\right) .
\end{gathered}
$$

let $t \longrightarrow 1$, then,

$$
\begin{gathered}
y_{1}(1)=x_{0}, \\
x_{1}(1)=x_{0} e^{\frac{-1}{\epsilon}}+\left(1-\rho x_{0} y_{1}\right)\left(1-e^{\frac{-1}{\epsilon}}\right) .
\end{gathered}
$$


For $t \in(1,2]$, take $x(t)=x_{1}=x_{1}(1), y_{1}(t)=y_{1}(1)=y_{1}$, when $t \leq 1$,

$$
\begin{gathered}
\text { then, } y_{2}(t)=x_{1}(t), \\
x_{2}(t)=x_{1} e^{\frac{-(t-1)}{\epsilon}}+\frac{1}{\epsilon} \int_{1}^{t} e^{\frac{s-t}{\epsilon}}\left(1-\rho x_{1} y_{1}\right) d s, \\
=x_{1} e^{\left.\frac{-(t-1}{\epsilon}\right)}+\left(1-\rho x_{1} y_{1}\right)\left(1-e^{\frac{-(t-1)}{\epsilon}}\right) .
\end{gathered}
$$

Let $t \longrightarrow 2$, then,

$$
\begin{gathered}
y_{2}(1)=x_{1}, \\
x_{2}(2)=x_{1}(1) e^{\frac{-1}{\epsilon}}+\left(1-\rho x_{1}(1) y_{1}(1)\right)\left(1-e^{\frac{-1}{\epsilon}}\right) .
\end{gathered}
$$

For $t \in(2,3]$, take $x(t)=x_{2}=x_{2}(2), y_{2}(t)=y_{2}(2)=y_{2}$, when $t \leq 2$,

$$
\begin{gathered}
\text { then, } y_{3}(t)=x_{2}(t), \\
x_{3}(t)=x_{1} e^{\frac{-(t-1)}{\epsilon}}+\frac{1}{\epsilon} \int_{2}^{t} e^{\frac{s-t}{\epsilon}}\left(1-\rho x_{2} y_{2}\right) d s \\
=x_{2} e^{\frac{-(t-2)}{\epsilon}}+\left(1-\rho x_{1} y_{2}\right)\left(1-e^{\frac{-(t-2)}{\epsilon}}\right) .
\end{gathered}
$$

Let $t \longrightarrow 3$, then,

$$
\begin{gathered}
y_{3}(3)=x_{2}, \\
x_{3}(3)=x_{2} e^{\frac{-1}{\epsilon}}+\left(1-\rho x_{2} y_{2}(1)\right)\left(1-e^{\frac{-1}{\epsilon}}\right) .
\end{gathered}
$$

Repeating the operation, we get that the solution of the system (10) is given by

$$
\begin{gathered}
y_{n+1}(t)=x_{n}(t), \\
x_{n+1}(t)=x_{n} e^{\frac{-(t-n)}{\epsilon}}+\left(1-\rho x_{n} y_{n}\right)\left(1-e^{\frac{-(t-n)}{\epsilon}}\right) .
\end{gathered}
$$

Let $t \longrightarrow n+1$, then,

$$
\begin{array}{r}
x_{n+1}=x_{n} e^{\frac{-1}{\epsilon}}+\left(1-\rho x_{n} y_{n}\right)\left(1-e^{\frac{-1}{\epsilon}}\right), \\
y_{n}=x_{n} .
\end{array}
$$


On the dynamics of the singularly perturbed Riccati differential equation...

\subsection{Local stability and bifurcation analysis of the discretized system}

The system (11) has two fixed points $\left(x_{1,2}^{*}, y_{1,2}^{*}\right)=\left(\frac{-1 \pm \sqrt{1+4 \rho}}{2 \rho}, \frac{-1 \pm \sqrt{1+4 \rho}}{2 \rho}\right)$.

Next, we calculate the Jacobian matrix at the first fixed point $\left(x_{1}^{*}, y_{1}^{*}\right)$

$$
J\left(x^{*}, y^{*}\right)=\left(\begin{array}{cc}
e^{\frac{-1}{\epsilon}}-\rho y^{*}\left(1-e^{\frac{-1}{\epsilon}}\right) & -\rho x^{*}\left(1-e^{\frac{-1}{\epsilon}}\right) \\
1 & 0
\end{array}\right) .
$$

Let us rename $-\rho x^{*}\left(1-e^{\frac{-1}{\epsilon}}\right)=z$, and $e^{\frac{-1}{\epsilon}}-\rho y^{*}\left(1-e^{\frac{-1}{\epsilon}}\right)=m$.

The characteristic equation

$$
\lambda^{2}-m \lambda-z=0
$$

has two roots

$$
\lambda_{1,2}=\frac{m \pm \sqrt{m^{2}+4 z}}{2} .
$$

Lemma 2. [9] Let $F(\lambda)=\lambda^{2}+P \lambda+Q$. Suppose that $F(1)>0$, and $F(\lambda)=0$ has two roots $\lambda_{1}$ and $\lambda_{2}$. Then

1. $F(-1)>0$ and $Q<1$ if and only if $\left|\lambda_{1}\right|<1$ and $\left|\lambda_{2}\right|<1$;

2. $F(-1)<0$ if and only if $\left|\lambda_{1}\right|<1$ and $\left|\lambda_{2}\right|>1\left(\right.$ or $\left|\lambda_{1}\right|>1$ and $\left.\left|\lambda_{2}\right|<1\right)$;

3. $F(-1)>0$ and $Q>1$ if and only if $\left|\lambda_{1}\right|>1$ and $\left|\lambda_{2}\right|>1$;

4. $F(-1)=0$ and $P \neq 0,2$ if and only if $\lambda_{1}=-1$ and $\left|\lambda_{2}\right| \neq 1$;

5. $P^{2}-4 Q<0$ and $Q=1$ if and only if $\lambda_{1}$ and $\lambda_{2}$ are complex and $\left|\lambda_{1,2}\right|=1$.

By applying (2), we get

$$
F(\lambda)=\lambda^{2}-m \lambda-z=\lambda^{2}+P \lambda+Q=0 .
$$

Then

$$
\begin{array}{r}
P=-m, \\
Q=-z .
\end{array}
$$


We have

$$
\begin{aligned}
& \qquad F(1)=1-m-z>0, \\
& \text { we should have } 1>m+z .
\end{aligned}
$$

By applying condition 1 of lemma (2)

$$
\begin{gathered}
\text { Where, } \quad F(-1)=1+m-z>0, \\
1+m>z \\
Q<1 \Rightarrow-z<1, z>-1 \\
\text { Where, }-\rho x^{*}\left(1-e^{\frac{-1}{\epsilon}}\right)=z
\end{gathered}
$$

substitute by the value of $x^{*}$, we get,

$$
\begin{gathered}
-\rho\left[\frac{-1+\sqrt{1+4 \rho}}{2 \rho}\right]\left(1-e^{\frac{-1}{\epsilon}}\right) \\
=\left(\frac{1-\sqrt{1+4 \rho}}{2}\right)\left(1-e^{\frac{-1}{\epsilon}}\right)>-1 .
\end{gathered}
$$

If (14) and (15) satisfied, then $\left(x_{1}^{*}, y_{1}^{*}\right)$ is stable.

The seconed fixed point is the same way of first fixed point.

\section{NUMERICAL SIMULATIONS}

We confirm all the previous analytical findings with the help of numerical simulations performed via Matlab. In all numerical simulations the initial condition is taken as $\left(x_{0}, y_{0}\right)=(0.4,0.4)$ and the Bif. parameter is taken as $\rho$.

We have the following examples 

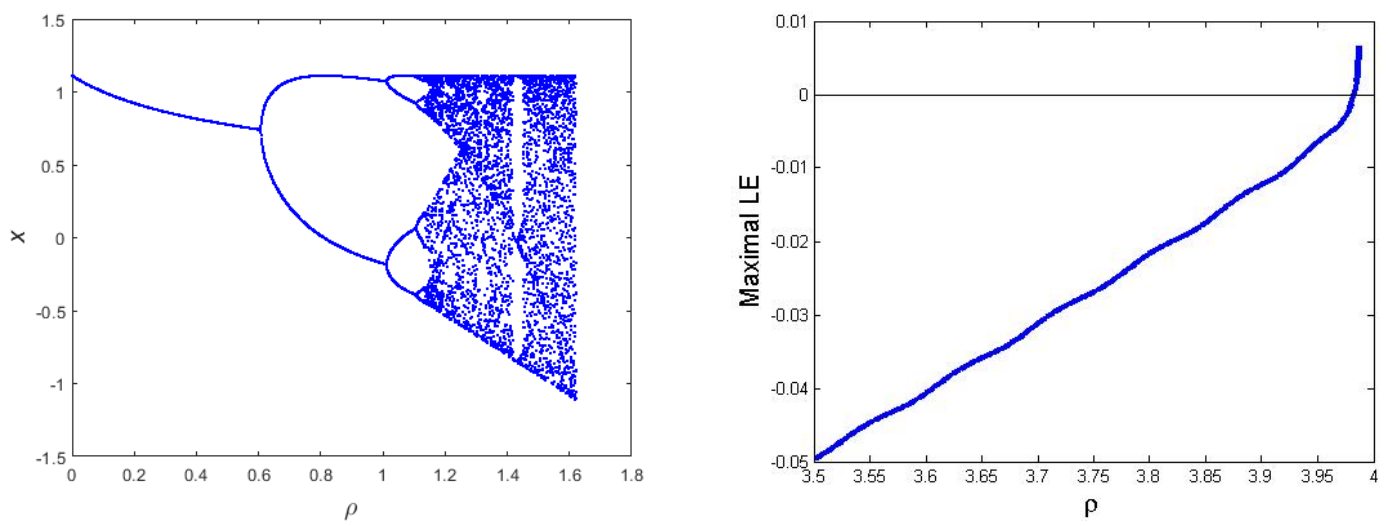

Figure 1: Bif.D. and the graph of Lyp.Ex. of system (11) as $\epsilon \longrightarrow 1$.

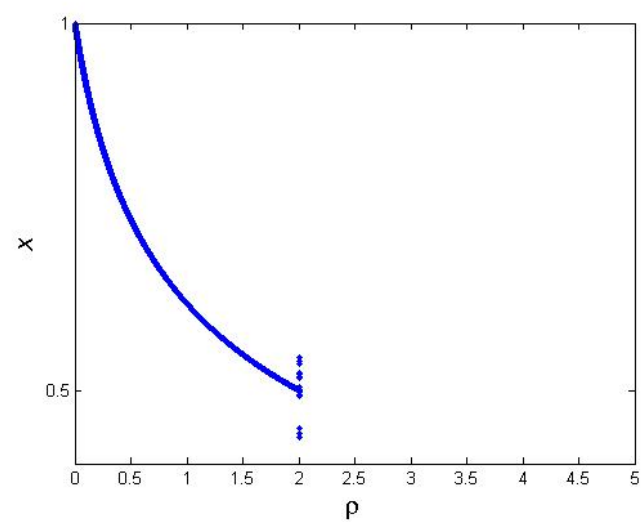

(a)

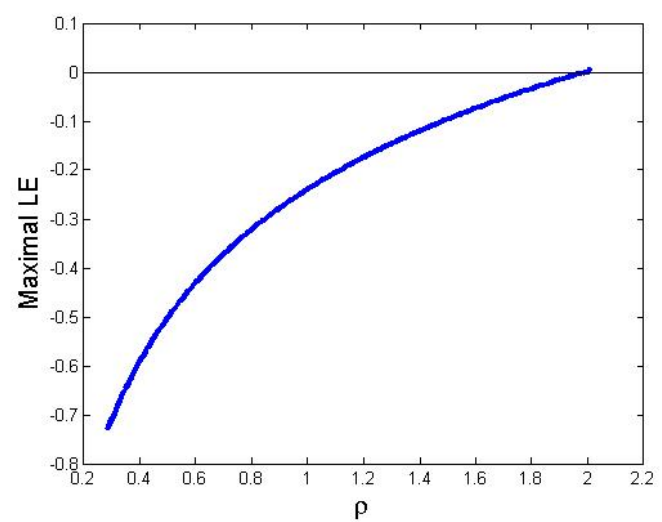

(b)

Figure 2: Hopf Bif.D. and the graph of Lyp.Ex. of system (11) as $\epsilon \longrightarrow 0$. 


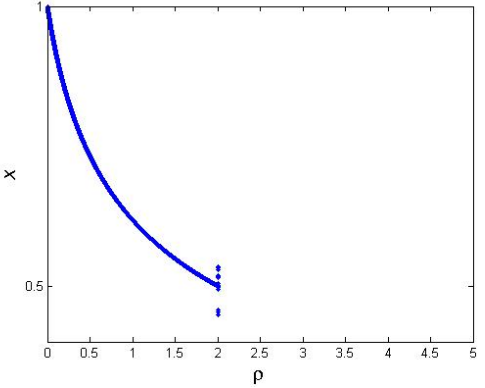

(a) $\epsilon=0.1$.

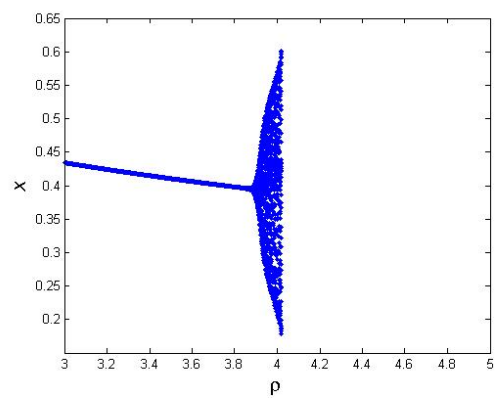

(c) $\epsilon=0.95$.

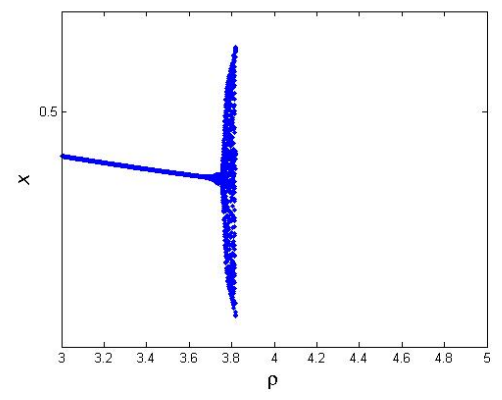

(e) $\epsilon=0.9$.

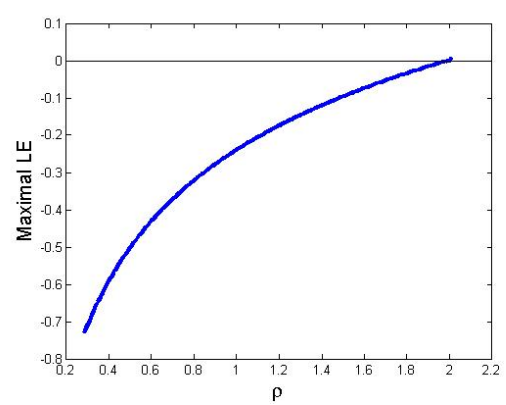

(b) $\epsilon=0.1$.

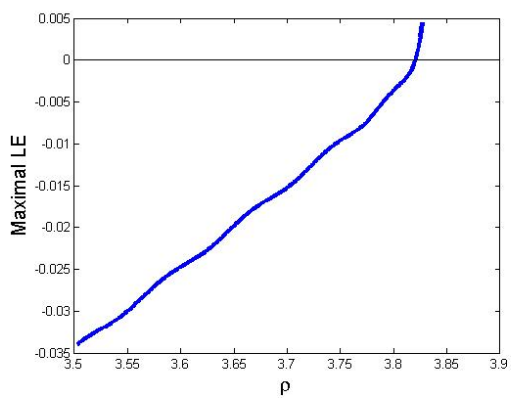

(d) $\epsilon=0.95$.

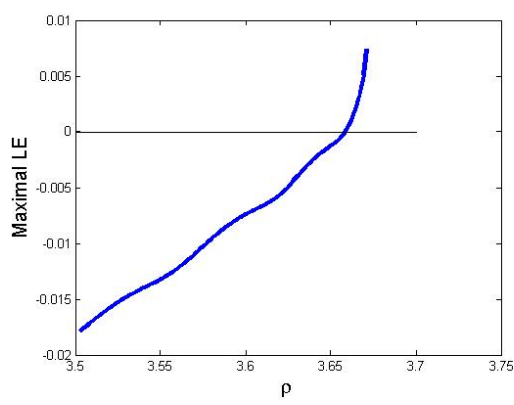

(f) $\epsilon=0.9$.

Figure 3: Hopf Bif.D. and the graph of Lyp.Ex. of system (11) for different values of $\epsilon$ 


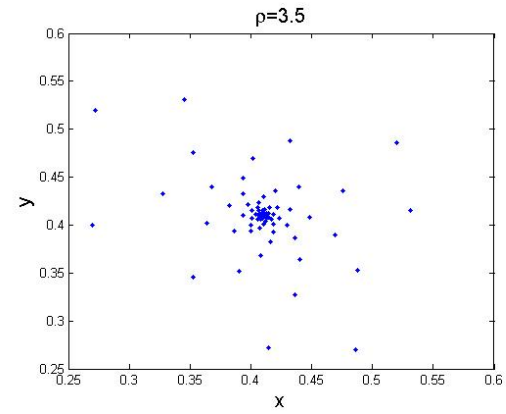

(a)

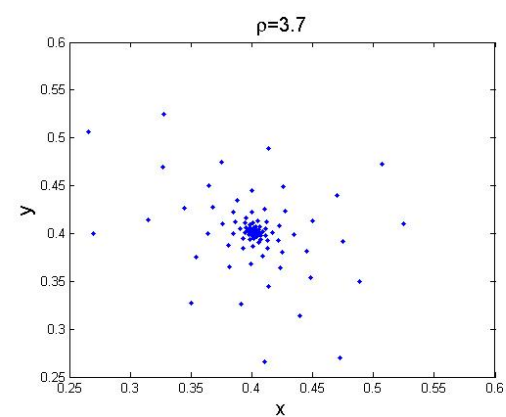

(c)

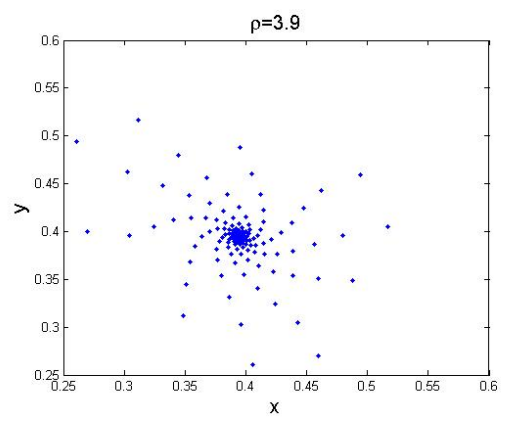

(e)

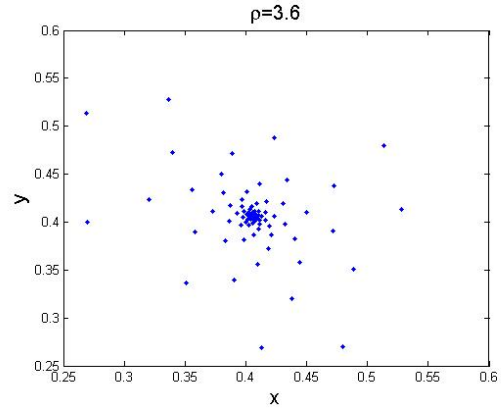

(b)

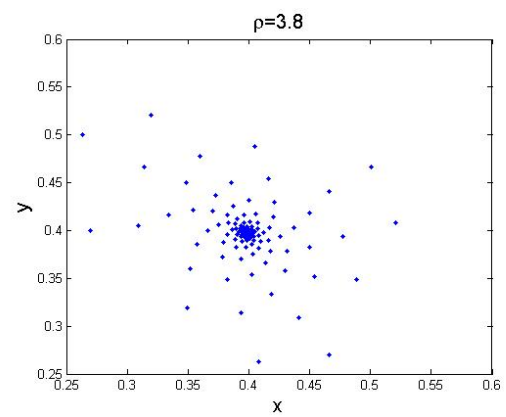

(d)

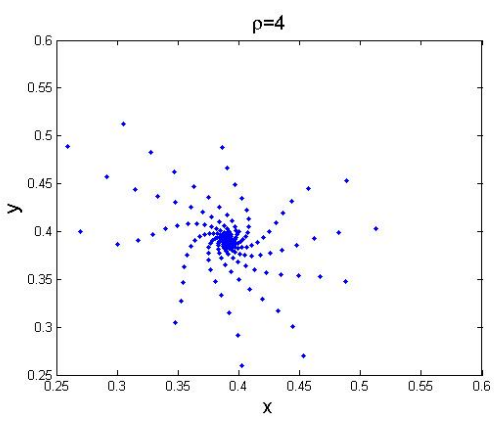

(f) 


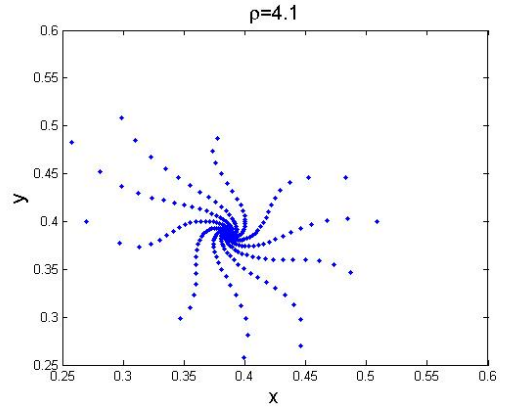

(g)

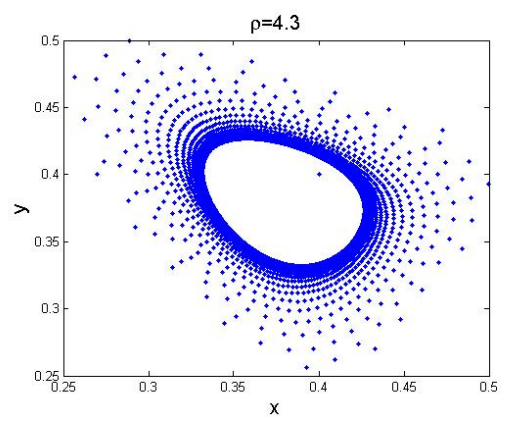

(i)

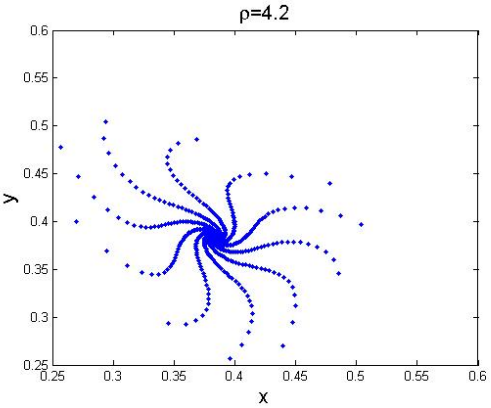

(h)

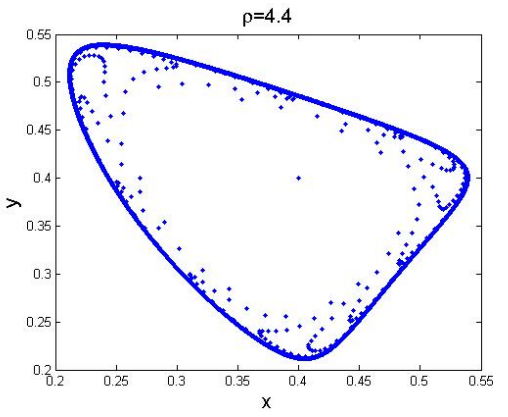

(j)

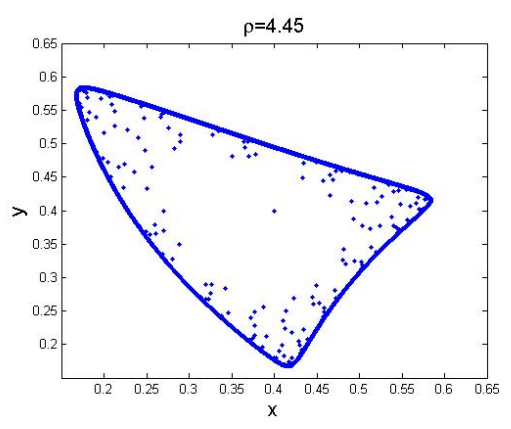

(k)

Figure 4: Phase portraits of system (11) for different $\rho$.

\section{CONCLUSION}

In this paper, we studied the dynamics of the S.P. Riccati difference equation with two different delays. First of all, we obtained F.P. and discussed their local stability by analyzing the corresponding characteristic equations of the linearized equations. secondly, we show that the equation exhibits Hopf Bif. and we have reached explicit conditions for its occurrence. Then, the method of steps is applied to obtain a discrete analogue of the considered system. We investigated local stability conditions of the F.P. of the discretized system. It is illustrated that the S.P. Riccati DDE with two delays 
behaves as the Riccati DDE with two delays. Finally, numerical simulations including Lyp. Ex., Bif.D. and ph.P.s carried out to confirm the theoretical analysis obtained and to illustrate more complex dynamics of the system.

\section{REFERENCES}

[1] Bellman,R. and Cooke,K. L. Differential-Difference Equations, Academic Press, New York, NY, USA, 1963.

[2] Driver,R. D. Ordinary and Delay Differential Equations, vol. 2 of Applied Mathematical Sciences, Springer, New York, NY, USA, 1977.

[3] McCartin

BJ. Exponential fitting of the delayed recruitment/renewal equation. Journal of Computational and Applied Mathematics. 2001 Nov 1;136(1-2):343-56.

[4] Tian H. The exponential asymptotic stability of singularly perturbed delay differential equations with a bounded lag. Journal of Mathematical Analysis and Applications. 2002 Jun 1;270(1):143-9.

[5] Glizer VY. Asymptotic analysis and solution of a finite-horizon H8 control problem for singularly-perturbed linear systems with small state delay. Journal of Optimization Theory and Applications. 2003 May 1;117(2):295-325.

[6] Ahmed, E., El-Sayed, A. M. A., El-Mesiry, A. E. M., \& El-Saka, H. A. A. (2005). Numerical solution for the fractional replicator equation. International Journal of Modern Physics C, 16(7), 1017-1026.

[7] Zhang B, Fan M. Near optimal control for singularly perturbed systems with small time-delay. In2008 7th World Congress on Intelligent Control and Automation 2008 Jun 25 (pp. 7212-7216). IEEE.

[8] Herzallah, M. A., El-Sayed, A. M., \& Baleanu, D. (2010). On the fractional-order diffusion-wave process. Rom. J. Phys, 55(3-4), 274-284.

[9] Albert, C. J. L. Regularity and complexity in dynamical systems. New York: Springer, 2012.

[10] EL-SAYED, A. M. A., et al. Dynamical analysis and circuit simulation of a new fractional-order hyperchaotic system and its discretization. International Journal of Bifurcation and Chaos, 2016, 26.13: 1650222.

[11] Elsonbaty, A. R., \& El-Sayed, A. M. A. (2017). Further nonlinear dynamical analysis of simple jerk system with multiple attractors. Nonlinear Dynamics, 87(2), 1169-1186. 
[12] El-Sayed, A. M. A., Salman, S. M., \& Elabd, N. A. (2018). On the dynamics of the singularly perturbed Mackey-Glass equation. Journal of Computational and Applied Mathematics, 344, 154-160.

[13] EL-SAYED,A. M. A., SALMAN,S. M. AND RAMADAN,S. DYNAMICAL ANALYSIS OF THE RICCATI DIFFERENTIAL EQUATION WITH DELAY, Advances in Mathematics: Scientific Journal 9 (2020), no.10, 1-15.

[14] EL-Sayed,A. M. A., Salman,S. M. and Ramadan,S. ON THE DYNAMICS OF THE SINGULARLY PERTURBED RICCATI DIFFERENCE EQUATION WITH CONTINUOUS ARGUMENT. Advances in Mathematics: Scientific Journal 10 (2021), no.2, 1-6.

[15] EL-Sayed,A. M. A., Salman,S. M. and Abo-Bakr,A. M. A. SINGULARLY PERTURBED LOGISTIC DIFFERENCE EQUATION. Advances in Mathematics: Scientific Journal 10 (2021), no.2, 943-948.

[16] EL-Sayed,A. M. A., Salman,S. M. and Abo-Bakr,A. M. A. On the dynamics of the logistic delay differential equation with two different delays, journal of applied and computional mechanics, 7(2)(2021), 442-449. 